\title{
Entropy and light cluster production in heavy-ion collisions at intermediate energies
}

\author{
Yogesh K. Vermani and Rajeev K. Pur** \\ Department of Physics, Panjab University, Chandigarh -160 014, India
}

(Dated: November 1, 2018)

\begin{abstract}
The entropy production in medium energy heavy-ion collisions is analyzed in terms of ratio of deuteronlike to protonlike clusters $\left(d_{\text {like }} / p_{\text {like }}\right)$ using quantum molecular dynamics (QMD) model. The yield ratios of deuteronlike-to-protonlike clusters calculated as a function of participant proton multiplicity closely agree with experimental trends. Our model predictions indicate that full thermodynamical equilibrium may not be there even for the central geometry. The apparent entropy extracted from the yield ratios of deuteronlike-to-protonlike clusters, however, reflects the universality characteristics i.e. it is governed by the volume of reaction independent of the target-projectile combination. Our calculations for apparent entropy produced in central collisions of $\mathrm{Ca}+\mathrm{Ca}$ and $\mathrm{Nb}+\mathrm{Nb}$ at different bombarding energies are in good agreement with $4 \pi$ Plastic Ball data.
\end{abstract}

\section{INTRODUCTION}

Various experimental and theoretical studies in the recent past have indicated a clear demarkation of colliding matter into participant and spectator matter especially at high incident energies [1, 2]. This is characterized by the formation of hot \& dense fireball. In addition, the size of the participant volume or 'source size' is also linked with the emission of composite particles and ultimately with the mechanism of the production of entropy [3 [5]. The highly dense fireball formed for a very short duration $\left(<10^{-22} s\right)$ can be of importance for probing the properties of condensed nuclear matter. The formation of the fireball is reported to be affected by many factors such as beam energy, overlapping volume, as well as density reached in a reaction. Earlier experimental studies using the Plastic Ball/Wall detector [6, 7] showed that the deuteron-to-proton yield ratio varies with the impact parameter of the reaction indicating a strong dependence on the participant volume. Such behavior is believed to be resulted from the coalescence production mechanism [6, 7]. Experimentally, the study of $d / p$ ratios obtained in asymmetric collisions of $\mathrm{p}+\mathrm{Kr}, \mathrm{O}+\mathrm{Kr}$, and $\mathrm{Ne}+\mathrm{Ar}$ also indicates the importance of coalescence mechanism [8]. In this study, it was observed that $d / p$ ratio increases with the beam energy. The baryonic entropy, however couldn't be directly measured from these ratios, since total yield is required from the source. The measurement of the yield ratios and baryonic entropy produced are, therefore, very promising candidates for estimating the fireball produced in the hot \& dense nuclear matter.

As a matter of fact, the pion production also contributes significantly [9 12 towards the entropy generation at SPS [13, 14] and higher energies. At SPS energies ( $160 \mathrm{AGeV}$ fixed target), the pion number increases with beam energy to about ten times the number of the nucleons [14]. There, an increase in the entropy production was observed with beam energy as one moves from AGS energies towards SPS and higher energies [10]. This enhancement may also be conjectured as manifestation of the change in the collision dynamics at such high energy [15, 16]. For the incident energy range considered (400-1050 AMeV) in the present work, the inclusion of the pion production is not going to affect the entropy production appreciably 11, 12, 17]. For instance, at SIS energies (upto 2 AGeV), the total number of pions is only $10 \%$ of the nucleons 18 .

In the present work, we aim to estimate the baryonic entropy $S_{N}$ generated in the fireball via the yields of free par-

*Electronic address: rkpuri@pu.ac.in 
ticles and light clusters (i.e. protons, neutrons, deuteron, tritium, helium-3, and $\alpha$-particles). We made a systematic study of symmetric heavy-ion (HI) collisions in the incident energy range 400-1050 AMeV within dynamical model namely, quantum molecular dynamics (QMD) model [19]. We shall also compare our results with experimental data taken with Plastic Ball/Wall detector. The dynamical approach such as QMD model provides an useful platform to estimate the yield of composite particles in terms of $n-n$ correlations from the start when the colliding nuclei are well separated to the final state where matter is cold and fragmented. As reported in Ref. [20], the production of light charged particles carries vital information about the stopping and equilibration of nuclear matter during a collision.

\section{THE QUANTUM MOLECULAR DYNAMICS MODEL}

The quantum molecular dynamics (QMD) model is a $n$-body transport theory that describes HI reactions in intermediate energy regime $\left(20 \mathrm{MeV} /\right.$ nucleon $\leq E_{l a b} \leq 2 \mathrm{GeV} /$ nucleon $)$ on event by event basis. The two essential ingredients of the model are nucleon-nucleon $(n-n)$ potential and stochastic scattering. Here each nucleon follows the trajectory according to the classical equations of motion [19]:

$$
\dot{\mathbf{p}}_{i}=-\frac{\partial\langle\mathcal{H}\rangle}{\partial \mathbf{r}_{i}}, \dot{\mathbf{r}_{i}}=\frac{\partial\langle\mathcal{H}\rangle}{\partial \mathbf{p}_{i}}
$$

The expectation value of the total Hamiltonian $\langle\mathcal{H}\rangle$ consists of kinetic and potential energies terms as:

$$
\begin{aligned}
\langle\mathcal{H}\rangle & =\langle\mathcal{T}\rangle+\langle\mathcal{V}\rangle \\
& =\sum_{i=1}^{A} \frac{\mathbf{p}_{i}^{2}}{2 m_{i}}+\sum_{i=1}^{A}\left(V_{i}^{\text {Skyrme }}+V_{i}^{Y u k}+V_{i}^{\text {Coul }}+V_{i}^{M D I}\right) .
\end{aligned}
$$

The $V_{i}^{\text {Skyrme }}, V_{i}^{Y u k}, V_{i}^{\text {Coul }}$ and $V_{i}^{M D I}$ in Eq.(2) are, respectively, the local Skyrme, long range Yukawa, an effective charge Coulomb and momentum dependent parts of the interaction [19]. The potential part without momentum dependent interactions (MDI) is called static equation of state (EoS). For the present study, we employ a soft equation of state represented by incompressibility $\kappa=200 \mathrm{MeV}$ and energy dependent $n$ - $n$ cross section [19]. The equation of state with MDI is labeled as 'SM' whereas without MDI, it is labeled as 'S'. Since many studies reveal the nuclear matter to be softer [21, 22], we also prefer the soft EoS.

The entropy information may be obtained from a classical charge symmetric gas of nucleons and deuterons in thermal and chemical equilibrium using the relation suggested by Kapusta et al. [3, 23].

$$
S_{N}=3.945-\ell n R_{d p}
$$

where $R_{d p}$ is the ratio of deuterons to protons established during early stages of the fireball. One source of error arises due to the neglect of other light composite particles viz. t, ${ }^{3} \mathrm{He}$ and $\alpha$. Bertsch and Cugnon [24] proposed to take into account these lighter fragments as well generalizing Eq.(3) as:

$$
S_{N}=3.945-\ln x
$$

where

$$
\begin{aligned}
x & =d_{\text {like }} / p_{\text {like }} \\
& =\frac{d+\frac{3}{2} t+\frac{3}{2}{ }^{3} H e+3 \alpha}{p+d+t+2{ }^{3} H e+2 \alpha} .
\end{aligned}
$$

As seen, quantity ' $x$ ' measures the yield ratio of deuteron-like $\left(d_{\text {like }}\right)$ to proton-like $\left(p_{\text {like }}\right)$ fragments. It has been well established from experiments that highest proton multiplicity accounts for most of the charges in HI system, 
thus leaving no room for heavier clusters. Following Ref. [25], we define the yield ratio of deuteron-like $\left(d_{l i k e}\right)$ to proton-like $\left(p_{\text {like }}\right)$ clusters in the following way:

$$
\tilde{R}_{d p}=\frac{Y(A=2)+\frac{3}{2} Y(A=3)+3 Y(A=4)}{N_{p}},
$$

where $\mathrm{Y}(\mathrm{A})$ stands for the number of fragments with mass ' $\mathrm{A}$ ' in one event. Analogous to experimental results, we calculate the total participant multiplicity $N_{p}$ as:

$$
\begin{gathered}
N_{p}=\frac{Z_{P}+Z_{T}}{A_{P}+A_{T}}[Y(A=1)+2 Y(A=2) \\
+3 Y(A=3)+4 Y(A=4)],
\end{gathered}
$$

where $Z_{P}+Z_{T}$ and $A_{P}+A_{T}$ define the total charge and mass of the colliding system. This procedure allows us to calculate the entropy produced in a reaction. We have calculated these ratios within QMD model for unfiltered events, employing minimum spanning tree (MST) procedure of clusterization [26]. In this clusterization approach, we assume that two nucleons sharing the same cluster if their centroids are closer than a spatial radius $r_{C}=\left|\mathbf{r}_{\alpha}-\mathbf{r}_{\beta}\right|$. One generally choose the radius $r_{C}=4 \mathrm{fm}$. It may be mentioned that some other sophisticated algorithms have also been developed which are based on relative momentum of nucleons [27], fragments' binding energy minimization [28], and backtracing procedure [29] to study the fireball and spectator matter physics.

\section{RESULTS AND DISCUSSION}

Since it is well established that the production of light charged particles and clusters, and ultimately the entropy is related to the fireball, it is of interest to see their phase space. In Fig. 1, we display the space (X-Z) of ${ }^{93} \mathrm{Nb}+{ }^{93} \mathrm{Nb}$ collision at $650 \mathrm{AMeV}$ and at a reduced impact parameter $b / b_{\max }=0.6$. The chosen time represents the freeze out time when density reaches asymptotic value. First of all, consistent with earlier attempts, we see that heavier fragments belong to the residue of either projectile or target, whereas lighter clusters like free nucleons and light charged particles LCPs $[2 \leq A \leq 4]$ are produced due to the coalescence and emerge from the mid-rapidity region. As shown in Ref. [20], these light charged particles carry vital information about the stopping as well as thermalization of the nuclear matter, therefore, are also good candidates for the study of production of entropy in HI reactions. A very little influence can be seen of momentum dependent interactions.

Next we study the final state composite particle yield ratios $\mathrm{X} / \mathrm{p}$ for the soft $(\mathrm{S})$ and soft momentum dependent (SM) interactions. This is shown in Fig. 2 for the collisions of ${ }^{93} \mathrm{Nb}+{ }^{93} \mathrm{Nb}$ at incident energy of $650 \mathrm{AMeV}$ as a function of impact parameter. Here ' $\mathrm{X}$ ' stands for $\mathrm{A}=2,3$ and 4 clusters. On can see that curves for different $\mathrm{X} / \mathrm{p}$ ratios exhibit similar trends:

(i) The $\mathrm{X} / \mathrm{p}$ ratio decreases with impact parameter (alternately, increases with $N_{P}$ ) indicating more production in central collisions compared to peripheral collisions. As shown by many authors [5, 6, 25], $N_{P}$ remains same for nearly central collisions and decrease sharply for semi-central collisions and peripheral collisions.

(ii) For central impact parameters (or, higher $N_{P}$ values), $\mathrm{X} / \mathrm{p}$ ratios reach an asymptotic value indicating that for central events, small variation in impact parameter does not give different results.

(iii) Role of momentum dependent interactions is nearly marginal justifying the earlier attempts [30] and use of soft equation of state.

Since entropy production is mostly measured for central collisions, the use of momentum dependent interactions will not give different results compared to static soft equation of state. These different yield ratios implied that one also obtains different behavior of density reached, collision rate and multiplicity of various light mass fragments for $\mathrm{S}$ and SM interactions. 
In Fig. 3, we extend the above study by including the ratio of deuteron-like $\left(d_{\text {like }}\right)$ to proton-like $\left(/ p_{\text {like }}\right)$ clusters. The calculations for $d_{\text {like }} / p_{\text {like }}$ are made using soft (S) equation of state for the collisions of ${ }^{40} \mathrm{Ca}+{ }^{40} \mathrm{Ca}$ (at 400 and $1050 \mathrm{AMeV}$ ) and ${ }^{93} \mathrm{Nb}+{ }^{93} \mathrm{Nb}$ (at 400 and $650 \mathrm{AMeV}$ ) as a function of participant proton multiplicity. The results of the Plastic Ball experiments [6] are also displayed for comparison. The Plastic ball data takes into account the overlap region for the yield of deuteronlike and protonlike clusters, while our ratios are calculated for the unfiltered events using MST procedure. The yield ratios are calculated typically after $40 \mathrm{fm} / \mathrm{c}$, when average nucleonic density saturates and $n$ - $n$ collisions practically cease. At this time, yields of composite particles is well established and may be compared with experimental data. One clearly sees that our model describes well the functional form of experimental $d_{\text {like }} / p_{\text {like }}$ ratio which is found to increase with $N_{P}$ (or centrality of the collision) and saturates at higher multiplicity end. At low $N_{P}$, there is a large drop in the yield ratio for model calculations as also found for individual clusterto-proton (X/p) ratios (See Fig.2). These trends are closely related with nuclear matter stopping and flow effect in the formation of hot and dense fireball. Recently, Dhawan et al. 20] studied impact parameter dependence of light charged particles (LCPs) yield and anisotropy ratio. It was found that LCPs production was maximum at central collisions where maximum stopping of nuclear matter is also achieved. Thus, production of light clusters can act an indicator of global stopping achieved in the nuclear matter. Interestingly, $d_{\text {like }} / p_{\text {like }}$ ratio calculated using dynamical approach is in good agreement with experimental data. This shows that one can reliably explore the applicability of dynamical approach such as QMD model to further investigate the formation of fireball at intermediate energies.

It should be emphasized that model of Kapusta [3, 4] is highly idealized one that relies on the assumption of thermal and chemical equilibrium in the system, for extraction of $S_{N}$ using Eqs. (3) and (4). The heavy-ion collisions at higher incident energies evolve from an initial non-equilibrium momentum distribution to a thermalized distribution. In a heavy-ion system, full equilibrium is not, however, always possible even at central geometry [31]. At large impact parameters, the momentum distribution of nucleons further deviates from the degree of full equilibrium [31]. It is worthwhile to study the equilibration of the nuclear matter produced at the time of fireball formation and role of secondary de-excitation of heavier clusters on proton-like and deuteron-like abundances during the expansion stage. The anisotropy ratio, which is also a measure of the degree of equilibration reached in a heavy-ion reaction, is defined as 20,31$]$ :

$$
\left\langle R_{A}\right\rangle=\frac{\sqrt{\left\langle p_{x}^{2}\right\rangle}+\sqrt{\left\langle p_{y}^{2}\right\rangle}}{2 \sqrt{\left\langle p_{z}^{2}\right\rangle}} .
$$

The full equilibrium corresponds to $\left\langle R_{A}\right\rangle$ value close to unity. In Fig. 4(a), we plot the time evolution of anisotropy ratio $R_{A}$ for the reactions of $\mathrm{Nb}+\mathrm{Nb}$ at incident energies of 400 and $650 \mathrm{AMeV}$, respectively. The shaded area corresponds to the time zone for highly excited nuclear matter which is followed by the decompression phase. Beyond this region, the nucleon density saturates and hard $n$ - $n$ collisions cease almost. One can see that $\left\langle R_{A}\right\rangle$ values approach close to asymptotic value just after the compression ( $\sim 40-45 \mathrm{fm} / \mathrm{c}$ ) irrespective of the incident energy chosen. The $\left\langle R_{A}\right\rangle$ values at both incident energies are still less than 1 . It clearly shows that the HI system has still not achieved full equilibrium at the expansion stage of the collision. It also indicates that $n$ - $n$ collisions occurring at later times don't alter the momentum space of nucleons significantly. In the lower panels (b)-(c), we display the yields of $p_{l i k e}$ and $d_{\text {like }}$ clusters. One can see that in low-density phase, the secondary emission from heavier clusters tend to populate the abundances of deuteron-like and proton-like clusters slightly.

Since full thermodynamical equilibrium is not visible for the participant zone, we shall estimate apparent entropy ' $S_{a p p}$ ' produced in the HI reactions using Eq.(44). We display in Fig. 5, the apparent entropy $S_{a p p}$ as a function of participant proton multiplicity $N_{P}$ for the reactions of $\mathrm{Ca}+\mathrm{Ca}, \mathrm{Nb}+\mathrm{Nb}$, and $\mathrm{Au}+\mathrm{Au}$ at incident energies of 400 and $650 \mathrm{AMeV}$. One can clearly see that at a given beam energy, it is the volume of participant nucleons (that is, $N_{P}$ ) which governs the apparent entropy produced rather than the total number of nucleons in the phase space. This clearly brings out the participant-spectator picture of HI collisions at relativistic beam energies. It depicts that 
participant volume is solely contributing towards deuteronlike and protonlike cluster abundances independent of the target-projectile combination. These results are in agreement with the experimental data and theoretical approaches. It may be mentioned that apparent entropy of the fireball would approach realistic baryonic entropy using Eq.(4) only when full thermodynamical equilibrium is achieved for the participant zone.

Finally we display in Fig. 6, the apparent entropy ' $S_{a p p}$ ' extracted for the central collisions of $C a+C a$ (at 400 and $1050 \mathrm{AMeV}$ ) and $N b+N b$ (at 400 and $650 \mathrm{AMeV}$ ). Also shown here is the baryonic entropy obtained in Plastic Ball experiment [6] using the model of Kapusta. Our model entropy values are in close agreement with experimental observations $[6,[32]$. Nearly no effect of beam energy is visible in experimental as well as in model entropies.

\section{SUMMARY}

In this paper, we have modeled the dynamical emission of light clusters in highly excited nuclear matter and estimated specific entropy based upon the formalism proposed by Kapusta et al. Our model calculations are done within the framework of quantum molecular dynamics (QMD) model for unfiltered events. The comparison of model calculations of yield ratios as a function of participant proton multiplicity $N_{P}$ with experimental data gives quite encouraging results. From these results, we conclude that it is the volume of participants in a reaction system that governs deuteronlike and protonlike cluster abundances in early interaction stage. The time evolution of momentum anisotropy ratio shows that full thermodynamical equilibrium isn't, however, achieved even at later expansion stage of the heavy-ion collisions. Our model calculations for apparent entropy produced in the central collisions of $\mathrm{Ca}+\mathrm{Ca}$ and $\mathrm{Nb}+\mathrm{Nb}$ indicate close agreement with experimental results based upon Kapusta's formalism. Nearly no effect of beam energy is visible in experimental as well as in model entropies.

[1] G. J. Kunde et al., Phys. Rev. Lett. 74 (1995) 38-41.

A. Schüttauf et al., Nucl. Phys. A 607 (1996) 457-486.

[2] B. de Schauenburg, F. Rami, and P. Wagner, GSI Scientific Reports 98-1 (1997) 56.

[3] P. J. Siemens and J. I. Kapusta, Phys. Rev. Lett. 43 (1979) 1486-1489.

[4] I. M. Mishustin, F. Myhrer and P. J. Siemens, Phys. Lett. B 95 (1980) 361-364.

L. P. Csernai and J. I. Kapusta, Phys. Rep. 131 (1986) 223-318.

[5] J. Aichelin and E. A. Remler, Phys. Rev. C 35 (1987) 1291-1296.

[6] K. G. R. Doss et al., Phys. Rev. C 32 (1985) 116-123.

[7] K. G. R. Doss et al., Phys. Rev. C 37 (1988) 163-168.

[8] A. Fokin et al., Phys. Rev. C 60 (1999) 024601-7.

[9] P. Braun-Munzinger, J. Stachel, J. P. Wessels, and N. Xu, Phys. Lett. B 344 (1998) 43-48.

[10] A. Andronic, P. Braun-Munzinger, and J. Stachel, Nucl. Phys. A 772 (2006) 167-199.

[11] L. Ahle et al., Phys. Rev. C 57 (1998) R466-R470.

[12] J. Barrette et al., Phys. Rev. C 62 (2000) 024901-8.

[13] P. Braun-Munzinger, I. Heppe, and J. Stachel, Phys. Lett. B 465 (1999) 15-20.

[14] S. V. Afanasiev et al., Phys. Rev. C 66 (2002) 054902-9.

[15] M. Gaździcki and D. Röhrich, Z. Phys. C 65 (1995) 215-223.

[16] M. Gaździcki, Z. Phys. C 66 (1995) 659-662.

[17] J. L. Klay et al., Phys. Rev. C 68 (2003) 054905-12.

[18] B. Hong et al., Phys. Lett. B 407 (1997) 115-120.

[19] J. Aichelin, Phys. Rep. 202 (1991) 233-360.

Ch. Hartnack et al., Eur. Phys. J A 1 (1998) 151-169. 
S. Kumar et al., Phys. Rev. C 58 (1998) 3494-3499.

J. Singh, S. Kumar, and R. K. Puri, Phys. Rev. C 62 (2000) 044617-8.

A. D. Sood and R. K. Puri, Phys. Rev. C 70 (2004) 034611-7.

A. D. Sood, R. K. Puri, and J. Aichelin, Phys. Lett. B 594 (2004) 260-264.

A. D. Sood and R. K. Puri, Phys. Rev. C 79 (2009) 064618-5.

Sanjeev Kumar, S. Kumar, and R. K. Puri, Phys. Rev. C 78 (2008) 064602-5.

[20] J. K. Dhawan, N. K. Dhiman, A. D. Sood, and R. K. Puri, Phys. Rev. C 75 (2006) 057901-4.

Sanjeev Kumar, S. Kumar, and R. K. Puri, Phys. Rev. C 81 (2010) 014601-7.

[21] F. Haddad et al., Phys. Rev. C 53 (1996) 1437-1439.

[22] S. Rosswog et al., Astronomy and Astrophysics 341 (1999) 499-526.

M. Itoh et al., Nucl. Phys. A 687 (2001) 52-57.

[23] L. P. Csernai and H. W. Barz, Z. Phys. A 296 (1980) 173-179.

L. P. Csernai, B. Lukacs, and J. Zimanyi, Nuovo Cimento Lett. 27 (1980) 111-116.

[24] G. Bertsch and J. Cugnon, Phys. Rev. C 24 (1981) 2514-2520.

G. Bertsch, Nucl. Phys. A 400 (1983) 221-232.

[25] G. Peilert, H. Stöcker, W. Greiner, A. Rosenhauer, A. Bohnet, and J. Aichelin, Phys. Rev. C 39 (1989) $1402-1419$.

[26] S. Kumar, R. K. Puri and J. Aichelin, Phys. Rev. C 58 (1998) 1618-1626.

J. Singh and R. K. Puri, J. Phys. G: Nucl. Part. Phys. 27 (2001) 2091-2108.

[27] J. K. Dhawan and R. K. Puri, Eur. Phys. J. A 33 (2007) 57-64.

[28] Y. K. Vermani and R. K. Puri, J. Phys. G: Nucl. Part. Phys. 36 (2009) 105103-8.

Y. K. Vermani, J. K. Dhawan, S. Goyal, R. K. Puri and J. Aichelin, J. Phys. G: Nucl. Part. Phys. 37 (2010) 015105-8.

Y. K. Vermani and R. K. Puri, Europhys. Lett. 85 (2009) 62001-6

R. K. Puri and J. Aichelin, J. Comput. Phys. 162 (2000) 245-266.

P. B. Gossiaux, R. K. Puri, Ch. Hartnack, and J. Aichelin, Nucl. Phys. A 619 (1997) 379-390.

R. K. Puri, Ch. Hartnack, and J. Aichelin, Phys. Rev. C 54 (1996) R28-R31.

C. O. Dorso and J. Aichelin, Phys. Lett. B 345 (1995) 197-202.

[29] J. P. Bondorf, D. Idier, and I. N. Mishustin, Phys. Lett. B 359 (1995) 261-267.

[30] J. Aichelin, A. Rosenhauer, G. Peilert, H. Stöcker, and W. Greiner, Phys. Rev. Lett. 58 (1987) $1926-1929$.

[31] D. T. Khoa et al., Nucl. Phys. A 542 (1992) 671-698.

[32] S. Nagamiya, M.-C. Lemaire, E. Moeller, S. Schnetzer, G. Shapiro, H. Steiner, and I. Tanihata, Phys. Rev. C 24 (1981) 971-1009. 


\section{Figure Captions}

FIG. 1. The snapshots of spatial coordinates of nucleons in $\mathrm{X}-\mathrm{Z}$ plane for the reaction of $\mathrm{Nb}(650 \mathrm{AMeV})+\mathrm{Nb}$ at reduced impact parameter $b / b_{\max }=0.6$. Time taken here corresponds to the case when $n$ - $n$ collisions cease almost just after the violent phase of the reaction. Different colors depict the free particles, nucleons bound in light charged particles as well as in heavier fragments.

FIG. 2. The yield ratio of light clusters to protons $(\mathrm{X} / \mathrm{p})$ as a function of impact parameter $\mathrm{b}$ using minimum spanning tree procedure. The term 'global' for ratios $\mathrm{X} / \mathrm{p}$ signifies that particle yield is calculated taking full ensemble into account and not the limited region only.

FIG. 3. The $d_{\text {like }} / p_{\text {like }}$ ratio as a function of baryon charge multiplicity $N_{p}$. The model calculations (open symbols) at the time of freeze out are compared with experimental data (solid symbols). The results are shown here for the reactions of $\mathrm{Ca}+\mathrm{Ca}$ (l.h.s.) and $\mathrm{Nb}+\mathrm{Nb}$ (r.h.s.).

FIG. 4. The anisotropy ratio $\left\langle\mathrm{R}_{A}\right\rangle$, and the yields of deuteron-like $\left(d_{\text {like }}\right)$ and proton-like $\left(p_{\text {like }}\right)$ clusters obtained during the evolution of central ${ }^{93} \mathrm{Nb}+{ }^{93} \mathrm{Nb}$ collisions at incident energies of 400 and $650 \mathrm{AMeV}$.

FIG. 5. Apparent entropy per nucleon $S_{a p p}$ as a function of baryon charge multiplicity $N_{p}$ for the reactions of ${ }^{40} \mathrm{Ca}+{ }^{40} \mathrm{Ca}$ (open circles), ${ }^{93} \mathrm{Nb}+{ }^{93} \mathrm{Nb}$ (half filled circles), and ${ }^{197} \mathrm{Au}+{ }^{197} \mathrm{Au}$ (open squares). Calculations shown here are at incident energies of 400(top) and 650(bottom) AMeV.

FIG. 6. The relationship between baryonic entropy and beam energy $E_{l a b}$ for the central collisions of ${ }^{40} \mathrm{Ca}+{ }^{40} \mathrm{Ca}$ and ${ }^{93} \mathrm{Nb}+{ }^{93} \mathrm{Nb}$. Also shown are entropy values extracted by the Plastic Ball group [6] using Kapusta's formalism. 


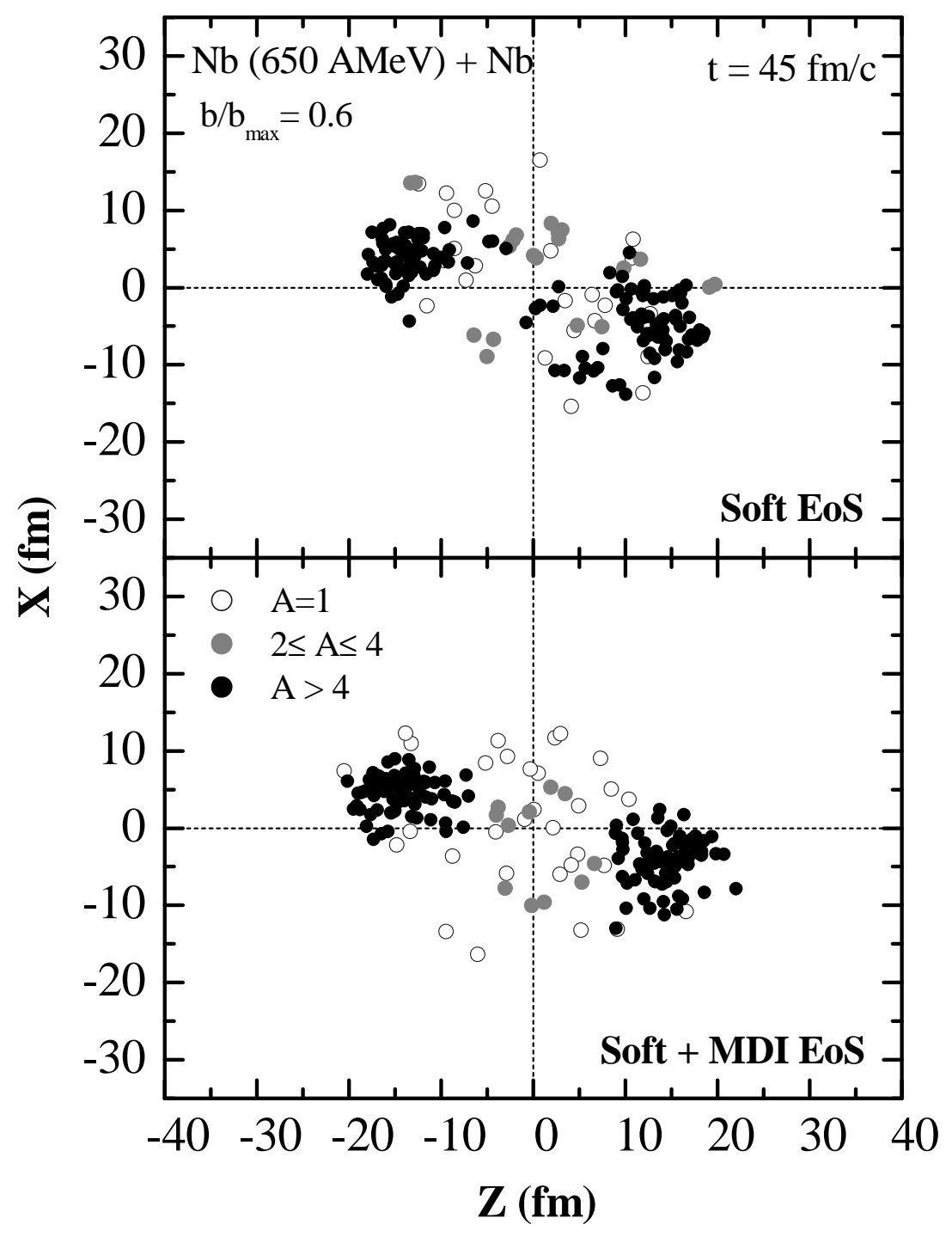

FIG. 1: 


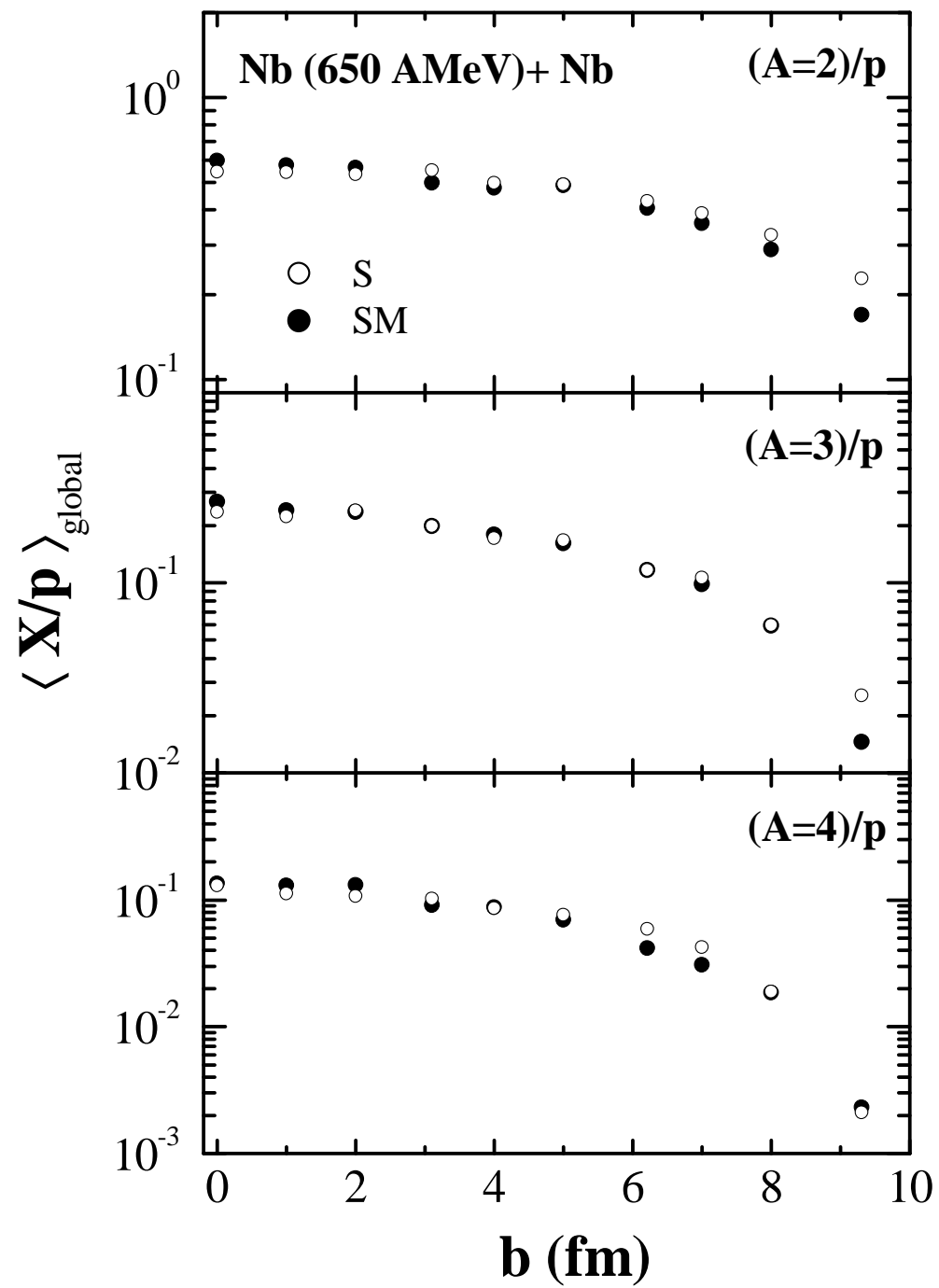

FIG. 2: 


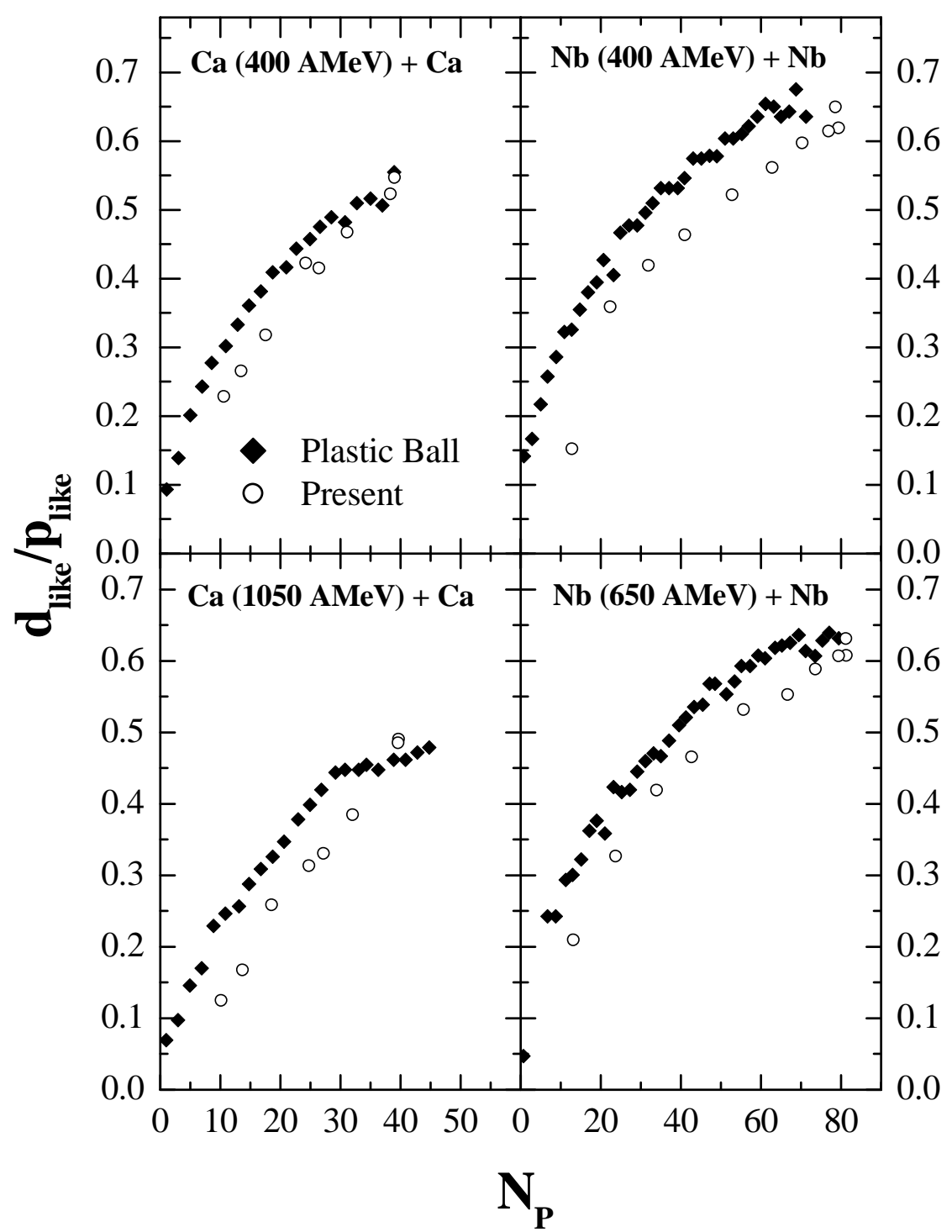

FIG. 3: 


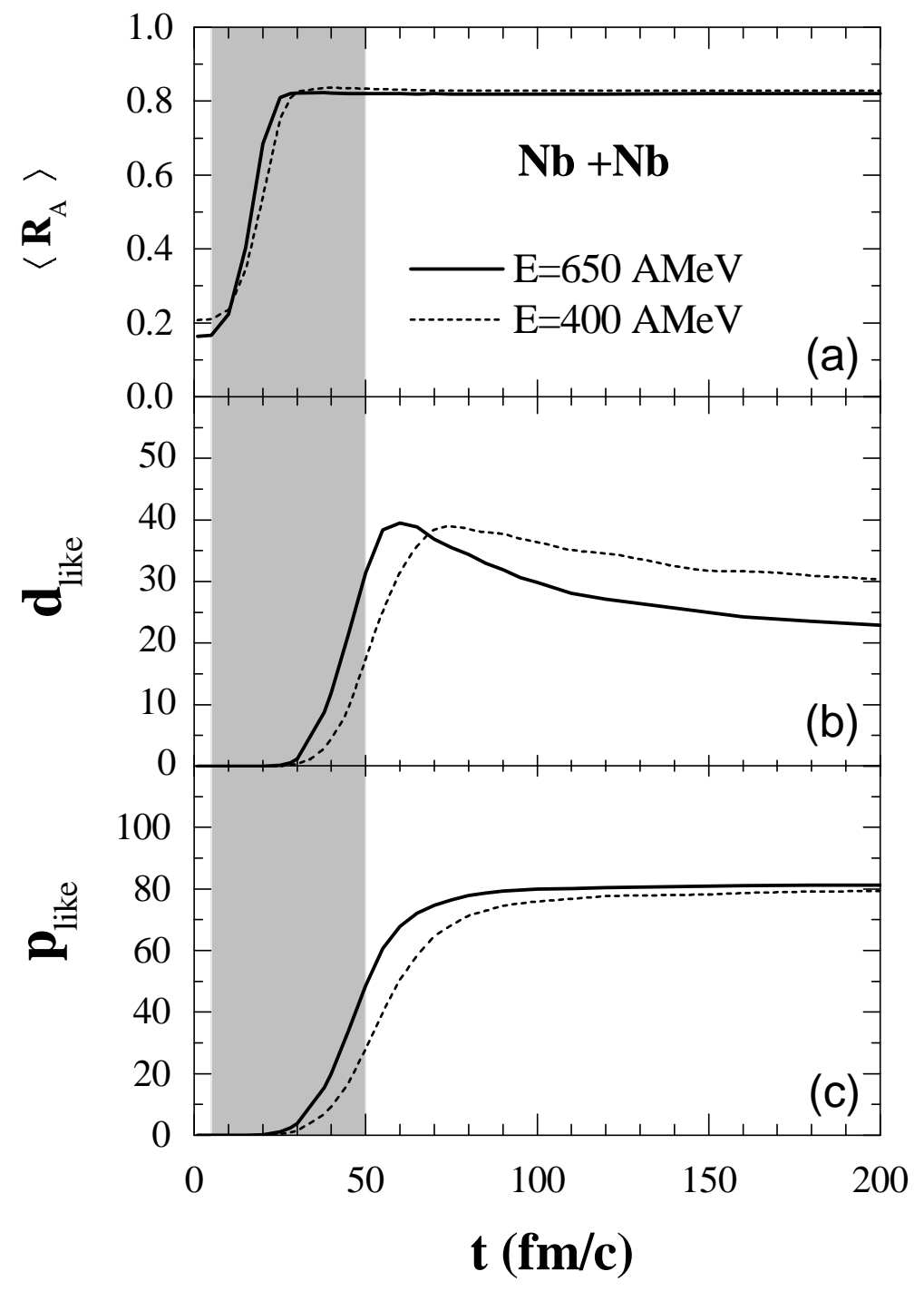

FIG. 4: 


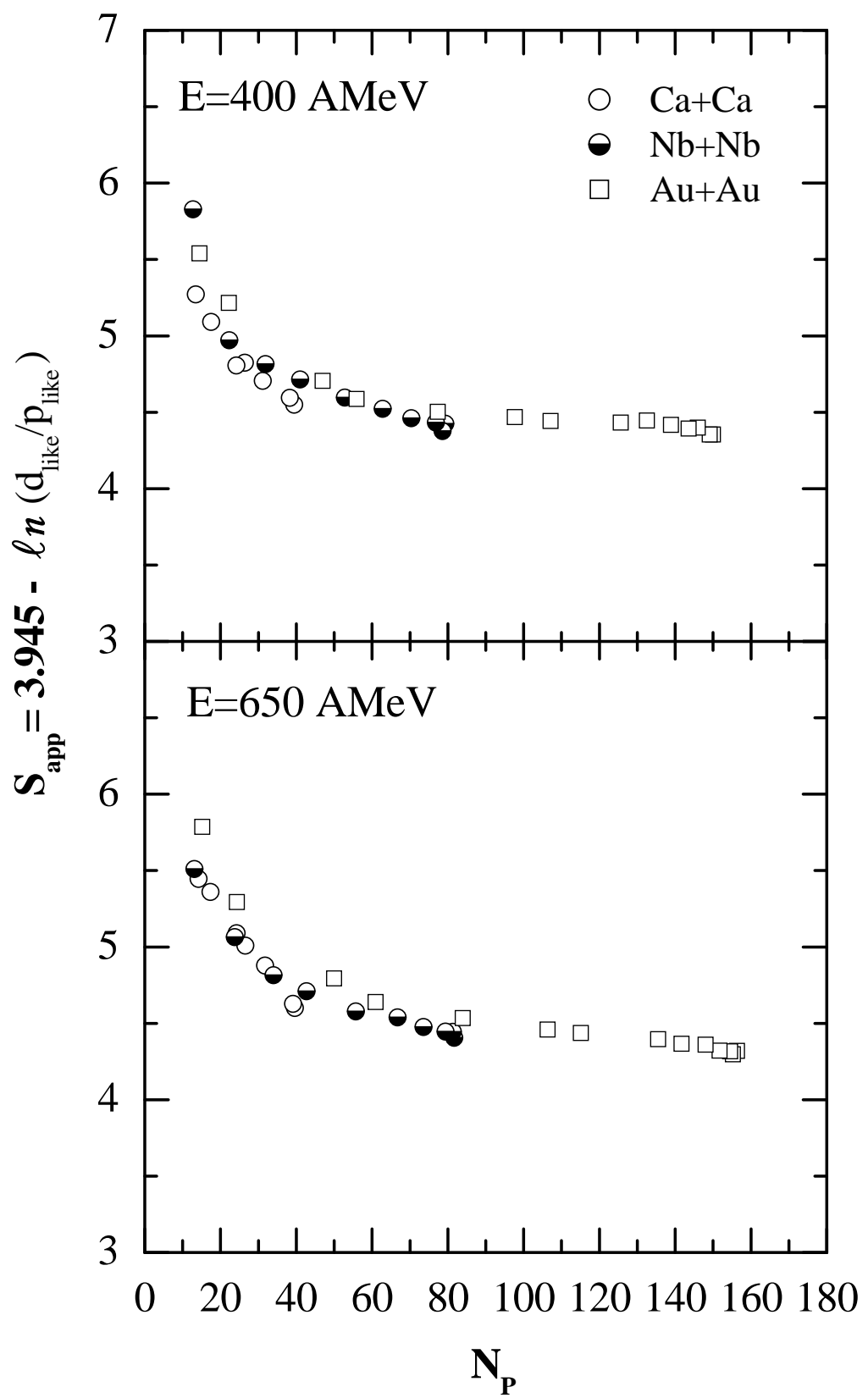

FIG. 5: 


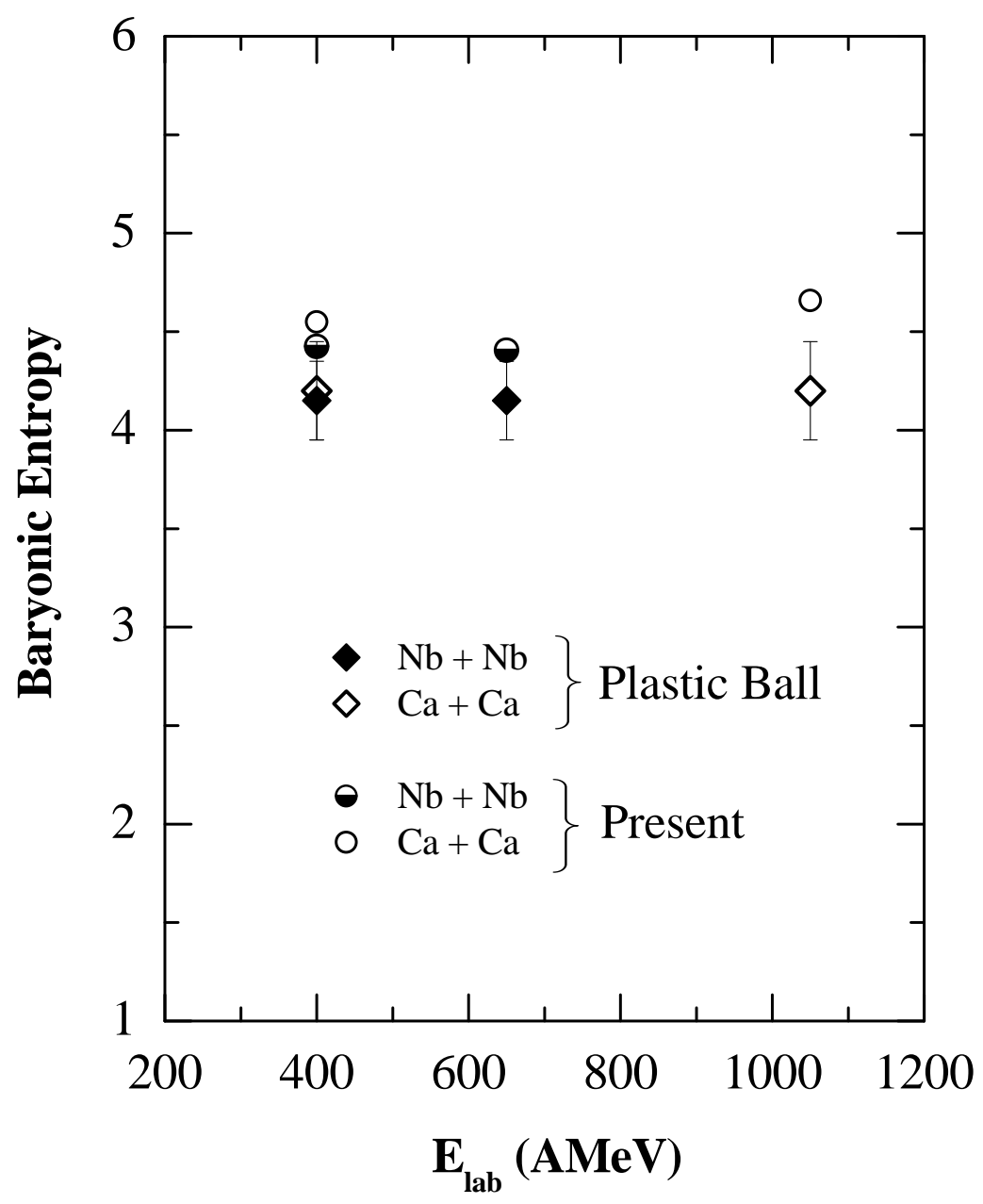

FIG. 6: 